\title{
An experimental approach to study the red blood cell dynamics in a capillary tube by biospeckle laser
}

\author{
Martin A. Toderi ${ }^{\mathrm{a}, \mathrm{b}}$, Bibiana D. Riquelme ${ }^{\mathrm{a}, \mathrm{b}}$, Gustavo E. Galizzi ${ }^{\mathrm{a}, \mathrm{c}, *}$ \\ ${ }^{a}$ Instituto de Física Rosario (CONICET-UNR), Bv. 27 de Febrero 210 bis, Rosario, \\ Argentina. \\ ${ }^{b}$ Facultad de Cs. Bioquímicas y Farmacéuticas, UNR, Suipacha 531, Rosario, Argentina. \\ ${ }^{c}$ Facultad de Cs. Exactas, Ingeniería y Agrimensura, UNR, Pellegrini 250, Rosario, \\ Argentina.
}

\begin{abstract}
In this study we investigated the human red blood cell (RBC) dynamics by means of biospeckle laser analysis. Blood samples from healthy donors were introduced in a $0.8 \mathrm{~mm}$ internal diameter capillary tube, and illuminated with a He-Ne laser in order to obtain the biospeckle pattern from both side and forward scattered light. Experiments were carried out for different concentrations of red blood cells in plasma, from $25 \%$ to $50 \%$. Biospeckle parameters such as Correlation Coefficient and Inertia Moment, were calculated for different frequency bandwidths in order to assess their sensitivity and versatility. A filter based on the Discrete Wavelet Transform was used to decompose the registered sample activity. A relation between Inertia Moment and the RBCs to plasma volume ratio was observed. The Correlation Index that measures the level of correlation of biospeckle images was defined and analyzed. This work inquires in a technique that is suitable for the development of novel non-invasive optical tools for clinical diagnosis in vascular pathologies.
\end{abstract}

Keywords: red blood cell, biospeckle laser, wavelet filtering, cell interaction

\footnotetext{
* Corresponding author

Email address: galizzi@ifir-conicet.gov.ar (Gustavo E. Galizzi)
} 


\section{Introduction}

Human red blood cell (RBC) interaction dynamics has a wide spectrum of interest, involving research on $\mathrm{RBC}$ mechanical properties [1, 2, 3, theoretical approaches [4, 5, 6, 7, optical and image processing techniques [8, 9, 10, 11] 5 and immediate application in Biomedicine [12, 13. Consequently, it is of great importance to develop techniques as well as equipment which allow the evaluation and characterization of blood dynamics due to its implications in vascular pathologies such as diabetes and hypertension [14, 15, 16. In particular, one of the main goals of these investigations is to contribute to the development of non-invasive techniques in order to early detect and monitor vascular diseases.

In vivo, $\mathrm{RBC}$ adhesion phenomena have a determinant role in blood flow and vascular resistance. One of the main consequences of the RBC interaction in stasis, i.e. without shear stress, is the formation of structures that normally consist of face-to-face linear assembles called rouleaux [17. Also, this phenomenon occurs simultaneously with the sedimentation of the structures due to gravity [18. RBC dynamics can be analyzed by several methods and techniques [19, 20, 21, 22, 23, 24] involving different sample volumes and previous preparation steps.

In the last few years, coherent light-based techniques have aroused attention due to their neglectable perturbation in the studied samples [25, 26]. When a scattering medium, e.g. a red blood cell suspension, is illuminated with a coherent light source, an interference pattern is observed at the image plane as shown in Fig. 1. This interferometric phenomenon carries information of the morphology of the illuminated sample. The final optical field is presented as a distribution of speckles, and when the distribution is time dependent, the phenomenon is called dynamic speckle. Moreover, if this variation in time is the consequence of a biological process, it is presented as biospeckle laser (BSL) [27, which has been observed as a sensitive way to study the dynamic behavior of blood [28, 29, 30, 31. Processes such as cell interaction, cytoplasmic fluctuations and biomechanical reactions related to water activity can be responsible for the 


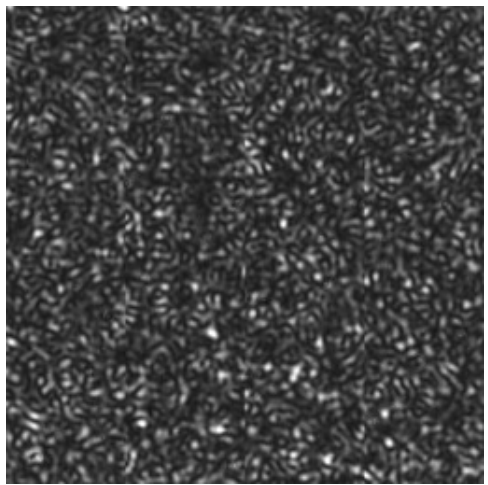

Figure 1: Typical speckle pattern obtained from a human blood sample.

variation of intensity of each speckle pattern.

In this work, BSL analysis was applied to study the dynamic behavior of different concentrations of RBCs inside a capillary tube, as a first approximation to the geometry of blood vessels. Determinations were performed obtaining

35 speckle patterns created by side and forward light scattering (SSC and FSC). In this way, we took into account that the interference phenomena develop differently due to the RBC motion and the different points of observation involved. In order to better identify the cell behavior, we applied a Discrete Wavelet Transform (DWT), which makes it possible to represent a signal through a reduced 40 number of factors 32. The fact that BSL is a non-destructive technique, together with the relatively simple experimental setup, could make it a relevant tool for biological applications.

\section{Materials and Methods}

\subsection{Blood Sample Preparation}

45 Whole blood samples were obtained from healthy donors $(\mathrm{n}=5)$ by venous puncture and anticoagulated with EDTA. Donor age range was from 25 to 30 years old (two women and three men), did not have any pre-existing health

problems (e.g. cardiovascular disease, respiratory disease, endocrine disease, 
haematological disorders or neoplasm etc.) and had normal hepatic, and renal function, coagulation and complete blood count. The samples were used within 4 hours after their extraction, according to the recommendations of Ref. [33]. RBCs were obtained from whole blood centrifuged for 5 minutes at $2000 \mathrm{rpm}$ (Paralwall Model PWL 12T). Buffy coat was discarded and plasma was separated and reserved for further use. RBCs were washed two times with saline solution. Samples were prepared with different ratios of RBCs to plasma volume (from $25 \%$ to $50 \%$ with steps of $5 \%$ ) mixing the washed RBCs with the autologous plasma. The RBCs concentration was checked by a Microhematocrit centrifuge (Rolco CH 24).

Each prepared blood sample was introduced in a $0.8 \mathrm{~mm}$ internal diameter capillary tube by means of a syringe just before conducting the experiment. RBCs interaction phenomenon occurrence was verified using a previously developed optical chip aggregometer [8, and the integrity of the cells was confirmed by optical microscopy.

\subsection{Experimental Setup}

The experimental setup is shown in Fig. 2. The light of a He-Ne laser ( $\lambda=632.8 \mathrm{~nm}, 60 \mathrm{~mW}$, Melles Griot) passed throughout an intensity dimmer and a 10x microscope lens (LE), which was used to expand the laser beam in order to uniformly illuminate the sample. Microscope lenses LS and LF, both $4 \mathrm{x}$ magnification, captured side and forward scattered beams. Two diaphragms were positioned after the lenses in order to control the biospeckle grain size. The sample was tightly placed in the optical path of both lenses slightly out of focus. Mirrors M1, M2 and M3 permitted the observation of the $90^{\circ}$ scattered beam that gives rise to the SSC biospeckle image. When M3 was removed, the FSC biospeckle image was obtained. Finally, experimental data were recorded 75 by a video camera (Dalsa model CA-D6, 256 by 256 pixels, $10 \mu \mathrm{m}$ per pixel) externally driven by a frame grabber (Coreco Imaging PC-DIG) and a CPU.

All experiments were conducted at controlled room temperature of $24^{\circ} \mathrm{C}$. Setup was mounted on a Melles Griot optical table to avoid any vibrational 


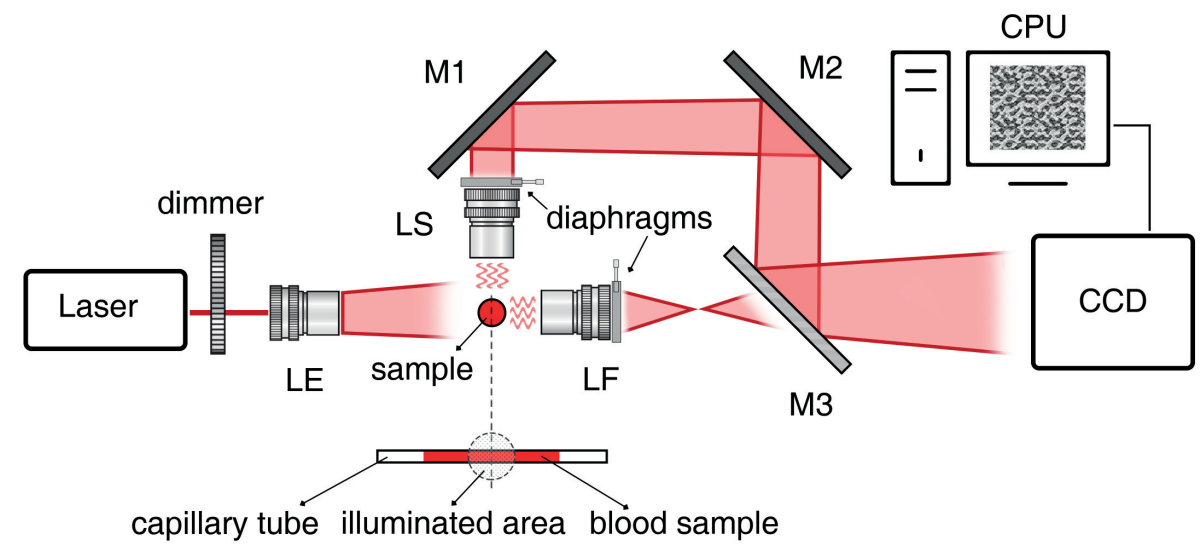

Figure 2: Schematic representation of the setup from the lateral side and showing a frontal view of the capillary tube containing the blood sample.

interference.

\subsection{Data Acquisition and Analysis}

The laser was turned on and left 10 minutes for beam stabilization. Camera and software were initialized and set to a frame rate of 8 frames per second. A 0.8 $\mathrm{mm}$ internal diameter capillary tube was previously filled with approximately 30 $\mu \mathrm{L}$ of blood and secured with syringes on both sides. After tightly positioning

${ }_{85}$ the sample, RBCs were slowly disaggregated as proposed in Ref. 34]. The recording of the biospeckle signal started 10 seconds after the disaggregation process ended, and the sample was left non perturbed throughout the entire recording process and 1600 uncompressed images were registered corresponding to a 200 s time lapse. By removing or repositioning mirror M3, two stacks of images were recorded per sample corresponding to FSC biospeckle and SSC biospeckle. The procedure was reproduced for each studied sample.

Data were analyzed by custom software as follows. Firstly, a 3D matrix containing the pixel intensity map for each frame was created. In order to better identify the information corresponding to RBC dynamic behavior, a quadrature mirror filter based on the Discrete Wavelet Transform (DWT) was applied to 
the recorded data. After signal decomposition, low frequencies and high frequencies were separated having the cut-off at 1/4 of the sampling frequency. Subsequently, the inverse process was performed but neglecting the low coefficients and the high coefficients respectively for each reconstruction. The final result consists of two 3D matrix containing the information from 0 to $\mathrm{f} / 4 \mathrm{~Hz}$ and $\mathrm{f} / 4$ to $\mathrm{f} / 2 \mathrm{~Hz}$ respectively, being $\mathrm{f}$ the sampling frequency.

\subsection{Biospeckle parameters}

Computational processing algorithms were applied to the original biospeckle signal and the filtered ones. The biospeckle parameters Correlation Coefficient (CC) and Inertia Moment (IM) were calculated in order to asses the RBC interaction phenomena.

The Correlation Coefficient $C C(k)$ between the first image and the subsequent ones is defined as 35]

$$
C C(k)=\frac{\langle I(0) I(k)\rangle-\langle I(0)\rangle\langle I(k)\rangle}{\left[\left(\left\langle I^{2}(0)\right\rangle-\langle I(0)\rangle^{2}\right)\left(\left\langle I^{2}(k)\right\rangle-\langle I(k)\rangle^{2}\right)\right]^{1 / 2}},
$$

where $k$ is the frame number that assumes values from $0,1, \ldots,(K-1)$ being $K$ the total number of images of the sequence recorded during the experiment. $I(k)$ is the pixel intensity map, a $2 \mathrm{D}$ matrix composed by the gray levels of each pixel corresponding to frame $k$, and \langle\rangle denotes the mean value operator, $i$. $e$. the mathematical average of the matrix elements. This coefficient provides a quantitative comparison of the similarity of each biospeckle pattern $I(k)$ of the temporal series with respect to the first one $I(0)$ chosen as the reference state. Moreover, the plot of the $C C(k)$ as a function of the frame number $k$ gives a quantitative measurement of the dynamics of the process under analysis [35. This index is related to the motion of the scatterers, in particular the RBCs, whose dynamics is expected to be conditioned by the number of cells present in the sample.

The computation of IM requires the previous calculation of the Time History Speckle Pattern (THSP) [36] and subsequently the Co-occurrence Matrix (COM) 37. The THSP shows the time evolution of a pixel intensity. In this 


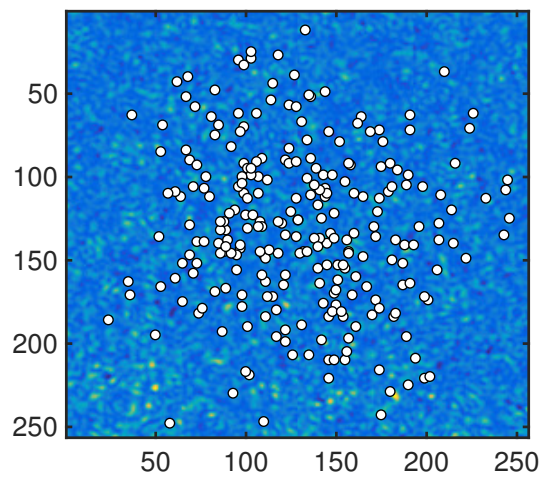

Figure 3: Gaussian distribution for 256 randomly chosen points around the center of the image (white circles), used to construct the THSP.

work, analyzed points were randomly selected based on a Gaussian distribution around the central pixel of the image (see Fig. 3 f for each calculation, giving a better statistical approach. Figure 4 shows a THSP used to calculate the corresponding COM. The COM evaluates the dispersion of consecutive pixels in the THSP, representing a transition histogram of intensity. The element $C O M(i, j)$ is the number of occurrences of a certain intensity value $i$ that is immediately followed by an intensity value $j$. Points are spreaded along the principal diagonal of the matrix as seen in Fig. 5 , indicating the activity of the sample. The aforementioned activity would be attributed to the Brownian motion of single cells, the formation of linear structures and the sedimentation process. Briefly, the higher the activity is, the wider the spread around the principal diagonal results.

The spread around the principal diagonal can be quantified by IM, which is defined as

$$
I M=\sum_{i} \sum_{j} \frac{\operatorname{COM}(i, j)}{N o r m}|i-j|^{2},
$$

where $i$ and $j$ are the matrix coordinates and Norm is the normalization of the co-occurrence matrix 38 . 


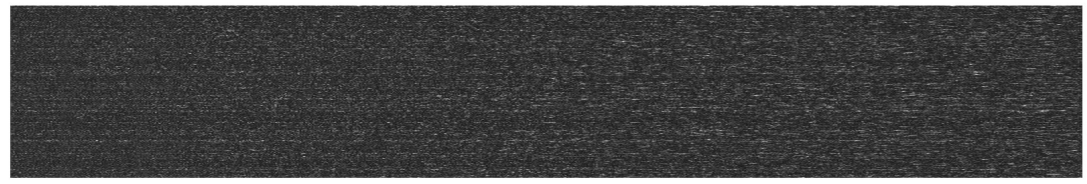

Figure 4: Time History Speckle Pattern (THSP) constructed from a Gaussian distribution of points for a $50 \%$ RBCs to plasma volume sample dynamics. Each row represents the time evolution of the intensity of the corresponding pixel.

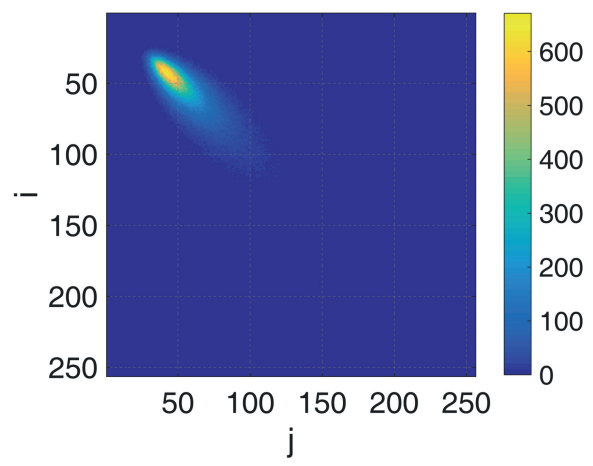

Figure 5: Co-occurrence Matrix (COM) calculated from the THSP presented in Fig 4 


\section{Results}

RBC interactions were studied by BSL analysis in samples of different ratios of RBCs to plasma volume. Variations of the parameters were observed and their sensitivity was evaluated obtaining the following results.

\subsection{Correlation Coefficient}

\subsection{Inertia Moment}

IM was calculated for different quantities of analyzed points, starting from 500 up to 10000 points with steps of 500 points as previously described. The 

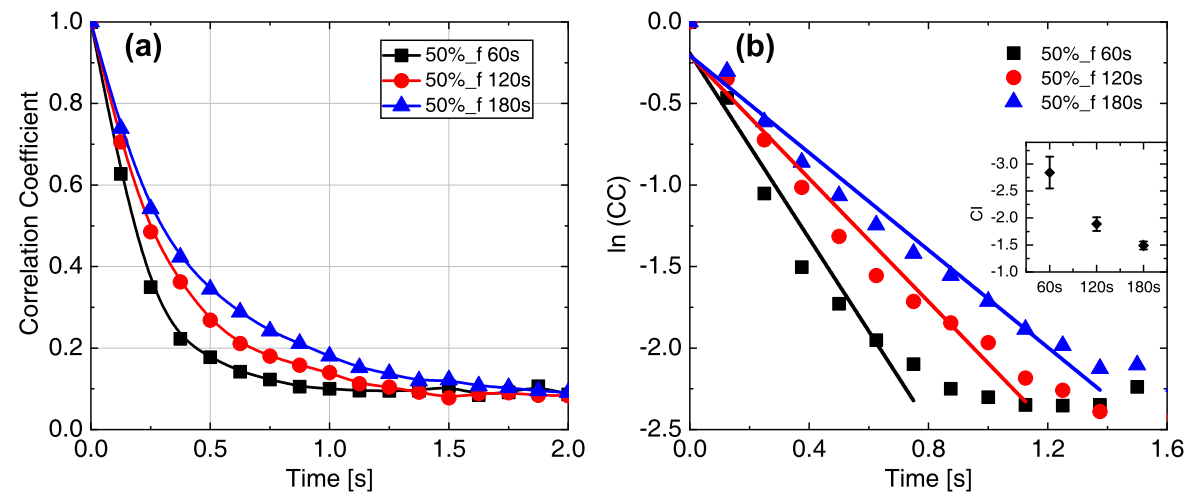

Figure 6: CC for FSC biospeckle data after DWT low pass filtering (a) and linearization of the $\mathrm{CC}$ (b) corresponding to $50 \%$ of RBCs to plasma volume, inset graphic shows the CI for every initial time.

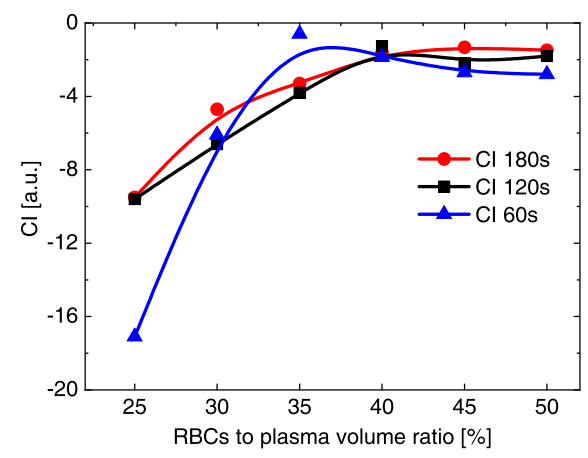

Figure 7: CIs calculated from FSC biospeckle data corresponding to different ratios of RBCs to plasma volume. The values correspond to the mean obtained from five samples for each ratio of RBCs to plasma volume. 
computing time was less than 2 minutes for the whole process per concentration per sample. Calculations were performed using the original signal. The IM values obtained from iterations with more than 4000 points of analysis showed insignificant variations for every $\mathrm{RBC}$ concentration. Consequently, this number of analyzed points was proposed for future calculations. Figure 8 (a) shows the IM value variation versus the number of analyzed points for the particular case of a $35 \%$ of RBCs to plasma volume sample. It can be noticed that after 4000 analyzed points the IM values tend to their mean value.

As the ratio of RBCs to plasma volume increases, light intensity is diminished due to a higher number of cells present in the sample. To address this issue, light intensity was adjusted to achieve the best contrast conditions regarding the compatibility speed between the camera and the phenomenon. Figure 8 (b) shows the mean light intensity versus time for four different ratios of RBCs to plasma volume. It can be noticed that the mean light intensity is approximately the same for every experiment and remains roughly constant with time throughout each measurement.

Figure 8 (c) and (d) depict the dependence of the IM on the RBC concentration present in each test. In the case of FSC biospeckle, the IM values are lower for higher number of cells but the tendency is reversed for SSC biospeckle. The reported IM values correspond to the mean obtained from five samples for each ratio of RBCs to plasma volume. 

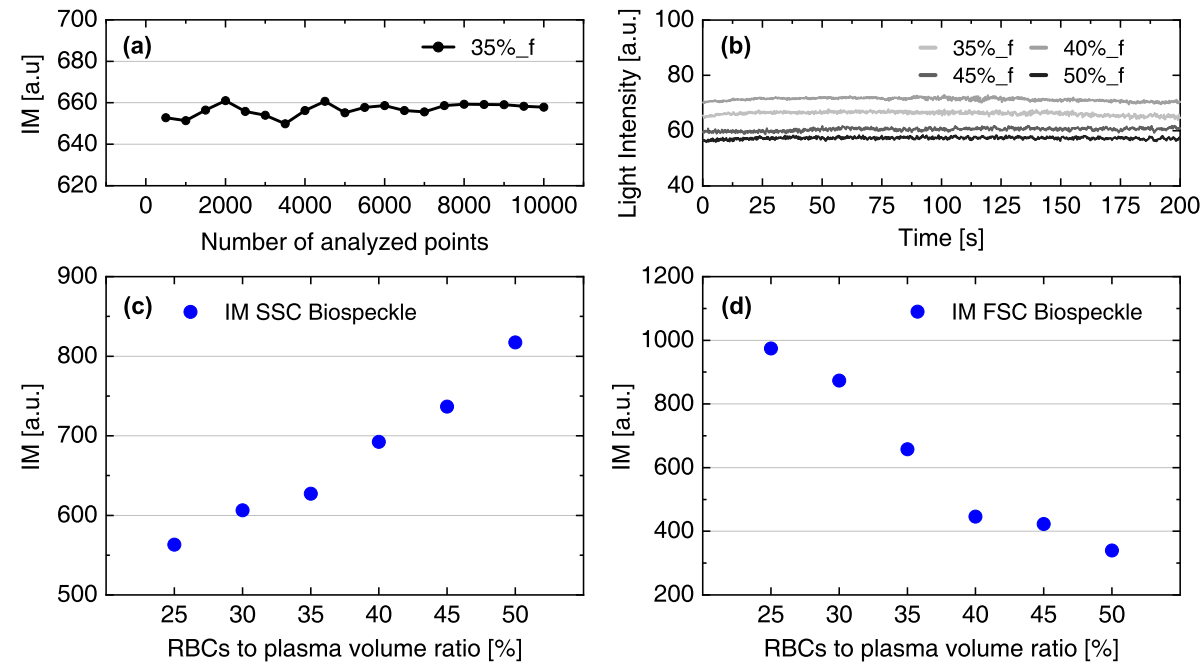

Figure 8: (a) IM values for several iterations of a random Gaussian distribution of selected points for a $35 \%$ RBCs to plasma volume concentration sample. (b) Mean light intensity during the measurement and for different RBCs to plasma volume ratios. IM values for different RBCs concentrations obtained for (c) FSC biospeckle and (d) SSC biospeckle.

\section{Discussion}

In this work a DWT based filter was applied to better distinguish RBC interactions, where blood samples were introduced in a capillary tube and illuminated with a He-Ne laser to obtain biospeckle images. Two arrangements were applied, SSC and FSC, obtaining the corresponding correlation coefficients for the high and low frequency bandwidth data.

This technique is based on collecting the random scattered light from the sample. Therefore, no geometrical reference is associated with the observed speckle pattern. The recorded information is the result of the contribution of all the particles that constitute the analyzed volume. The speckle grains are consequence of constructive and destructive interference of the scattered light, thus there is no actual visualization of the RBCs. Consequently, this technique does not distinguish inhomogeneities in the particle density inside the capillar related to geometrical aspects. In addition, eventual fluctuations of 
the hematocrit inside the tube would be masked by the global behavior of the the mean intensity is not determined by each specific sample characteristics. This fact would make it possible to use the obtained IM vs. hematocrit curves without the requirement of initial calibration while preserving the biospeckle 


\section{Conclusion}

In the present work we studied the human RBC dynamics by means of biospeckle laser analysis, emulating the geometry of a vessel with a capillary tube. It was possible to obtain two kinds of biospeckle images from one experimental setup corresponding to the SSC and FSC by simply repositioning one mirror. It is presumable that the $\mathrm{RBC}$ interaction activity information is mainly contained in the low frequency bandwidth determined by DWT filtering of the data obtained by forward scattering biospeckle. The IM parameter could be used as a RBCs to plasma volume identifier. medium were performed. Therefore, further studies would involve the alteration of the aggregation properties of the RBCs and the protein content of the plasma, both determinant factors in blood viscosity and microcirculation. The simplicity of the experimental arrangement together with the low-cost components required and its potential non-invasive attribute makes the biospeckle method stand out among the coherent light-based techniques. These characteristics make it suitable for assessing erythrocyte dynamics to improve diagnosis and further treatment of vascular pathologies, in particular because of the statistical nature and time dependency of the results. Short term objectives concern

\section{Acknowledgments}

Authors thank Dr. Mabel D'Arrigo director of the Clinical Analytical Chemistry Laboratory of the FCByF (UNR) and Dr. Anala Alet for the collaboration 
and direction in the preparation of biological samples. This work was partially supported by grant PI BIO400 from UNR and grant PIP 11220150100607 from

\section{References}

[1] E. Evans, R. Hochmuth, Membrane viscoelasticity, Biophysical Journal 16 (1) (1976) 1 - 11. doi:10.1016/S0006-3495(76)85658-5.

[2] B. Riquelme, J. Valverde, R. Rasia, Complex viscoelasticity of normal and lectin treated erythrocytes using laser diffractometry, Biorheology 35 (4) (1998) 325 - 334. doi:10.1016/S0006-355X (99)80014-6.

[3] B. D. Riquelme, J. R. Valverde, R. J. Rasia, Determination of the complex viscoelastic parameters of human red blood cells by laser diffractometry, Proc. SPIE, Optical Diagnostics of Biological Fluids V 3923 (2000). doi: $10.1117 / 12.387133$

[4] E. Kaliviotis, M. Yianneskis, Blood viscosity modelling: influence of aggregate network dynamics under transient conditions, Biorheology 48 (2) (2011) 127-47.

[5] S. K. Doddi, P. Bagchi, Three-dimensional computational modeling of multiple deformable cells flowing in microvessels, Phys. Rev. E 79 (2009) 046318. doi:10.1103/PhysRevE.79.046318.

[6] F. Janoschek, F. Toschi, J. Harting, Simplified particulate model for coarsegrained hemodynamics simulations, Phys. Rev. E 82 (2010) 056710. doi: 10.1103/PhysRevE.82.056710.

[7] T. Ye, N. Phan-Thien, C. T. Lim, L. Peng, H. Shi, Hybrid smoothed dissipative particle dynamics and immersed boundary method for simulation of red blood cells in flows, Phys. Rev. E 95 (2017) 063314. doi: 10.1103/PhysRevE.95.063314. 
[8] M. A. Toderi, H. Castellini, B. Riquelme, Descriptive parameters of the erythrocyte aggregation phenomenon using a laser transmission optical chip, Journal of Biomedical Optics 22 (1) (2017) 017003/1-8. doi: 10.1117/1.JBO.22.1.017003.

[9] G. Popescu, Y. Park, W. Choi, R. R. Dasari, M. S. Feld, K. Badizadegan, Imaging red blood cell dynamics by quantitative phase microscopy, Blood Cells, Molecules, and Diseases 41 (1) (2008) 10 - 16. doi:10.1016/j. bcmd.2008.01.010.

[10] G. Jiao, R. Zhang, Modeling of micropipette aspiration and optical tweezers stretching of erythrocytes with or without malaria parasite, Theoretical and Applied Mechanics Letters 3 (3) (2013) 034001. doi:10.1063/2.1303401.

[11] E. Kaliviotis, J. M. Sherwood, S. Balabani, Partitioning of red blood cell aggregates in bifurcating microscale flows, Scientific Reports 7 (2017) 44563. doi:10.1038/srep44563.

[12] S. Shin, Y. Yang, J.-S. Suh, Measurement of erythrocyte aggregation in a microchip stirring system by light transmission, Clinical Hemorheology and Microcirculation 41 (3) (2009) 197-207.

[13] P. Ponce de Leon, M. Toderi, H. Castellini, B. Riquelme, In vitro alterations of erythrocyte aggregation by action of trichinella spiralis newborn larvae,

口 Clinical Hemorheology and Microcirculation 65 (2) (2017) 195-204. doi: $10.3233 / \mathrm{CH}-16158$.

[14] B. Riquelme, M. D'Arrigo, P. Foresto, R. Rasia, Laser diffractometry technique for determination of stationary and dynamics viscoelastic parameters of erythrocyte in vascular pathologies, Proc. SPIE, Optical Coherence Tomography and Coherence Techniques 5140 (2003). doi:10.1364/ECBO. 2003.5140_229.

315 [15] M. Delannoy, A. Fontana, M. D'Arrigo, B. Riquelme, Influence of hyperten- 
sion and diabetes mellitus on erythrocyte aggregation using image digital analysis, Series on Biomechanics 29 (1) (2015) 5-10.

[16] Y. I. Cho, M. P. Mooney, D. J. Cho, Hemorheological disorders in diabetes mellitus, Journal of diabetes science and technology 2 (6) (2008) 11301138. doi:10.1177/193229680800200622

[17] S. Chien, L. A. Sung, Physicochemical basis and clinical implications of red cell aggregation, Clinical Hemorheology 7 (1987) 71-91.

[18] T. Fabry, Mechanism of erythrocyte aggregation and sedimentation, Blood 70 (5) (1987) 1572-1576.

[19] O. K. Baskurt, H. Meiselman, E. Kayar, Measurement of red blood cell aggregation in a plateplate shearing system by analysis of light transmission, Clinical Hemorheology and Microcirculation 19 (4) (1998) 307-314.

[20] R. Rasia, N. de Isla, L. Altube, J. Stoltz, J. Valverde, Determination of adhesive specific energy of erythrocyte agglutination by laser retrodiffusion, Optics and Lasers in Engineering 39 (5) (2003) 599 - 607. doi:10.1016/ S0143-8166(02) 00051-9.

[21] C. Lacombe, J. Lelivre, Interpretation of rheograms for assessing rbc aggregation and deformability, Clinical Hemorheology and Microcirculation 7 (1987) 46-61.

335 [22] S. Chen, G. Barshtein, B. Gavish, Y. Mahler, S. Yedgar, Monitoring of red blood cell aggregability in a flow-chamber by computerized image analysis, Clinical Hemorheology and Microcirculation 14 (4) (1994) 497-508.

[23] M. Boynard, J. Lelievre, R. Guillet, Aggregation of red blood cells studied by ultrasound backscattering, Biorheology 24 (5) (1987) 451-461.

${ }_{340}$ [24] R. M. Bauersachs, R. B. Wenby, H. J. Meiselman, Determination of specific red blood-cell aggregation indexes via an automated-system, Clinical Hemorheology and Microcirculation 9 (1989) 1-25. 
[25] K. Basak, G. Dey, M. Mahadevappa, M. Mandal, D. Sheet, P. K. Dutta, Learning of speckle statistics for in vivo and noninvasive characterization of cutaneous wound regions using laser speckle contrast imaging, Microvascular Research 107 (2016) 6 - 16. doi:10.1016/j.mvr.2016.04.008

[26] T. Kyoden, S. Naruki, S. Akiguchi, H. Ishida, T. Andoh, Y. Takada, N. Momose, T. Homae, T. Hachiga, In vivo visualization method by absolute blood flow velocity based on speckle and fringe pattern using two-beam multipoint laser doppler velocimetry, Journal of Applied Physics 120 (8) (2016) 084701. doi:10.1063/1.4961611.

[27] H. J. Rabal, R. A. Braga Jr. (Eds.), Dymanic Laser Speckle and Applications, CRC Press, 2008.

[28] Y. Aizu, T. Asakura, Bio-speckle phenomena and their application to the evaluation of blood flow, Optics \& Laser Technology 23 (4) (1991) 205-219. doi:10.1016/0030-3992(91)90085-3.

[29] R. Farraro, O. Omid Fathi, B. Choi, Handheld, point-of-care laser speckle imaging, Journal of Biomedical Optics 21 (2016) 21-21-6. doi:10.1117/ 1.JBO.21.9.094001

[30] S. M. White, R. Hingorani, R. P. Arora, C. C. Hughes, S. C. George, B. Choi, Longitudinal in vivo imaging to assess blood flow and oxygenation in implantable engineered tissues, Tissue Engineering Part C: Methods 18 (9) (2012) 697-709. doi:10.1089/ten.tec.2011.0744.

[31] Y. Piederriere, J. L. Meur, J. Cariou, J. Abgrall, M. Blouch, Particle aggregation monitoring by speckle size measurement; application to 『 blood platelets aggregation, Opt. Express 12 (19) (2004) 4596-4601. doi: 10.1364/OPEX.12.004596.

[32] M. Vetterli, C. Herley, Wavelets and filter banks: theory and design, IEEE 口 Transactions on Signal Processing 40 (9) (1992) 2207-2232. doi:10.1109/ 78.157221 . 
[33] O. K. Baskurt, M. Boynard, G. C. Cokelet, P. Connes, B. M. Cooke, S. Forconi, F. Liao, M. R. Hardeman, F. Jung, H. J. Meiselman, G. Nash, N. Nemeth, B. Neu, B. Sandhagen, S. Shin, G. Thurston, J. L. Wautier, New guidelines for hemorheological laboratory techniques, Clinical Hemorheology and Microcirculation 42 (2) (2009) 75-97. doi:10.3233/ CH-2009-1202.

[34] O. K. Baskurt, M. Uyuklu, S. Ozdem, H. J. Meiselman, Measurement of red blood cell aggregation in disposable capillary tubes, Clinical Hemorheology and Microcirculation 47 (4) (2011) 295-305. doi:10.3233/CH-2011-1411

[35] A. Federico, G. H. Kaufmann, G. E. Galizzi, H. Rabal, M. Trivi, R. Arizaga, Simulation of dynamic speckle sequences and its application to the analysis of transient processes, Optics Communications 260 (2) (2006) 493-499. doi:10.1016/j.optcom.2005.11.047.

[36] A. Oulamara, G. Tribillon, J. Duvernoy, Biological activity measurement on botanical specimen surfaces using a temporal decorrelation effect of laser

口 speckle, Journal of Modern Optics 36 (2) (1989) 165179. doi:10.1016/ S0030-3992(99) 00033-X

[37] R. Arizaga, M. Trivi, H. Rabal, Speckle time evolution characterization by the co-occurrence matrix analysis, Optics \& Laser Technology 31 (2) (1999) 163 - 169. doi : 10.1016/S0030-3992(99)00033-X.

[38] R. Cardoso, R. Braga, Enhancement of the robustness on dynamic speckle laser numerical analysis, Optics and Lasers in Engineering 63 (2014) 19-24. doi:10.1016/j.optlaseng.2014.06.004.

[39] R. Braga, G. Horgan, A. Enes, D. Miron, G. Rabelo, J. Barreto Filho, Biological feature isolation by wavelets in biospeckle laser images, Computers

a and Electronics in Agriculture 58 (2) (2007) 123-132. doi:10.1016/j. compag.2007.03.009 
[40] R. A. Braga, L. Dupuy, M. Pasqual, R. R. Cardoso, Live biospeckle laser imaging of root tissues, European Biophysics Journal 38 (5) (2009) 679- 